\title{
Mild Obstructive Sleep Apnea Tends to Be Associated with Sleep State Misperception
}

\author{
Joon-Ho Lee', ${ }^{1,2}$, Sehyun Jeon ${ }^{1,2}$, and Heon-Jeong Lee ${ }^{1,2,3}$ \\ 'Department of Psychiatry, Korea University College of Medicine, Korea University Anam Hospital, Seoul, Korea \\ ${ }^{2}$ Chronobiology Institute, Korea University, Seoul, Korea \\ ${ }^{3}$ Department of Biomedical Science, Korea University College of Medicine, Seoul, Korea
}

\begin{abstract}
Objective: Sleep state misperception (SSM) is a disorder that is characterized by insomnia or excessive sleepiness without objective evidence of sleep disturbance. The cause of SSM has not yet been elucidated. This study aimed to explore sleep perception tendencies by comparing polysomnography (PSG) data and subjective sleep reports in patients with and without obstructive sleep apnea (OSA). Methods: We retrospectively analyzed 638 participants with PSG results. We classified the participants into three groups based on their OSA severity (non-OSA, $n=174$; mild OSA, $n=214$; moderate OSA, $n=250$ ), and compared the PSG parameters and various sleep questionnaires among the groups using an analysis of variance. Additionally, we identified the rapid eye movement (REM)-related OSA and compared the variables between the REM-related OSA and non-REM-related OSA groups using independent $t$ and chi-square tests. Results: The participants with less severe OSA presented better quality and quantity of sleep in PSG (longer total sleep time, higher sleep efficiency, fewer arousals, and more deep sleep), but reported much worse sleep disturbances based on sleep questionnaires. Those with REM-related OSA showed a larger discrepancy between subjective and objective sleep. Conclusion: This study found that individuals with milder OSA and REM-related OSA tended to present larger discrepancies in objective and subjective sleep states. Further research based on our preliminary findings is warranted to better recognize SSM and treat complicated sleep disorders.
\end{abstract}

Key Words: Obstructive sleep apnea; Paradoxical insomnia; REM-related obstructive sleep apnea; Sleep misperception

Received: August 12, 2020 Revised: August 30, 2020 Accepted: August 31, 2020

Corresponding author: Sehyun Jeon, MD, Department of Psychiatry, Korea University College of Medicine, Korea University Anam Hospital, 73 Goryeodae-ro, Seongbuk-gu, Seoul 02841, Korea.

Tel: 82-2-920-6269, E-mail: shiskool@gmail.com

(a) This is an Open Access article distributed under the terms of the Creative Commons Attribution Non-Commercial License (https://creativecommons.org/licenses/bync/4.0) which permits unrestricted non-commercial use, distribution, and reproduction in any medium, provided the original work is properly cited.

\section{INTRODUCTION}

Insomnia is a prevalent sleep disorder that is diagnosed primarily based on subjective sleep perception. A considerable proportion of patients with insomnia tend to exaggerate their poor sleep quality $[1,2]$. Sleep state misperception (SSM) or paradoxical insomnia refers to the marked mismatch between objective findings such as polysomnography (PSG) and subjective self-report [3].

Patients with SSM usually overestimate sleep latency and wakefulness after sleep onset (WASO) and underestimate the total sleep time and the amount of deep sleep [4]. They also over-report the consequences of insomnia in daytime functioning, such as fatigue and negative mood. In contrast, other patients with sleep disorders overestimate the total sleep duration, which some researchers term positive sleep discrepancy [5]. Either phenomenon can signifi- cantly affect the patient's quality of life, have clinical consequences, and cause difficulties in setting treatment goals. The diagnostic criteria and prevalence of SSM are diverse and inconsistent. A systematic review reported 19 different definitions, with the prevalence ranging from $14 \%$ to $64 \%$ [6].

There have been various contributing factors to SSM suggested. Among the patient factors, Dorsey and Bootzin [7] demonstrated that the scores of personality dimensions, particularly in neuroticism and introversion, are related to subjective sleep tendencies and sleep perception. Vanable et al. [8] reported that current psychopathology, such as depressive disorders, can affect subjective sleep. Sleep electroencephalogram (EEG) characteristics have also been shown to be associated with sleep perception. Feige et al. [9] reported that significant hyperarousal and sleep microstructural differences contribute to SSM and chronic insomnia. 
A recent study by Hermans et al. [10] using sleep EEG suggested that sleep fragmentation in the early part of the night sleep contributes to the mechanisms of SSM. The proportion of each sleep stage [i.e., N3 stage sleep, rapid eye movement (REM) sleep] is also suggested as an important factor affecting SSM [11-13]. In particular, REM sleep is suggested to contribute to subjective sleep perception through increased cortical activity by two studies $[11,12]$.

Obstructive sleep apnea (OSA) is a common sleep breathing disorder manifested by increased arousal and sleep fragmentation, which overlap with candidate etiologies of SSM $[9,10]$. Patients with OSA often experience arousal at the end of apnea and hypopnea, and experience sleep fragmentation due to repetitive respiratory events that disturb deep sleep. Poor night sleep quality in patients with OSA causes daytime sleepiness and fatigue $[14,15]$. Therefore, we can assume that OSA is an important contributing factor to the development of SSM mediated by respiratory eventrelated arousal and sleep fragmentation.

Only a few studies have been conducted to explore the relationship between OSA and sleep perception. In a retrospective study based on PSG data, participants with OSA were found to significantly underestimate their total sleep time and overestimate sleep latency compared to those without OSA [13]. In another study, a considerable sleep mismatch was observed in patients with OSA [16]. In contrast, Pinto et al. [17] reported that sleep-disordered breathing did not contribute to different sleep perception, while patients with insomnia perceived poorer sleep quality compared to the normal control. Bianchi et al. [18] suggested that sleep perception cannot be explained by objective PSG measures of fragmentation in patients with OSA, presenting an absence of correlation between subjective sleep duration and the objective sleep fragmentation index, such as the arousal index and sleep efficiency. Choi et al. [19] suggested that patients with OSA have different PSG profiles based on the presence or absence of subjective insomnia complaints. Therefore, a differentiation between the clinical subtypes is needed to tailor the treatment in OSA.

Thus, the findings on the association between OSA and sleep perception are inconsistent. Moreover, all of these previous studies were conducted with small sample sizes. Therefore, further large-scale studies are needed to better elucidate the characteristics of sleep perception in patients with OSA.

Based on previous studies, we hypothesized that OSA would account for some of the SSM in an individual. In this study, we aimed to explore sleep perception tendencies by retrospectively evaluating PSG and subjective sleep report data in a large clinical sample. We compared the PSG parameters and subjective sleep scores among the different OSA severity groups. In addition, we investigated whether sleep perception tendencies differ depending on sleep stage-specific OSA characteristics performing subgroup analyses in OSA participants of both genders and in those of male.

\section{METHODS}

\section{Participants}

We recruited participants who underwent PSG between January 1, 2015, and December 31, 2019, at the Sleep Center of Korea University Anam Hospital. The exclusion criteria were as follows: age $<18$ years, incomplete PSG or questionnaire data, extreme PSG findings such as severe OSA [apnea-hypopnea index (AHI) $\geq 30$ ], and total sleep time $<100 \mathrm{~min}$. Due to the wide range of AHI and heterogeneity of probable medical comorbidity in severe OSA, we excluded the severe OSA to specifically focus subjective sleep perception. In total, 638 participants were included and classified into three groups based on the severity of OSA (non-OSA, mild OSA, and moderate OSA). The study protocol was approved by the Institutional Review Board (IRB) of the Korea University Anam Hospital (IRB No. 2020AN0177).

\section{Polysomnography}

All participants underwent nocturnal PSG performed with RemLogic soft wear and a device (Embla, Broomfield, CO, USA). Data were recorded using 6-channel electroencephalography, 2-channel electrooculography, chin electromyography, and electrocardiogram. Experienced PSG technicians performed and scored the PSG based on the American Academy of Sleep Medicine recommendations [20].

\section{Diagnostic criteria}

OSA was diagnosed based on the PSG results. The criteria for OSA followed the International Classification of Sleep Disorders (3rd edition) [3]. The severity of OSA was defined according to the AHI value (mild, $5 \leq \mathrm{AHI}<15$; moderate, $15 \leq \mathrm{AHI}<30$ ). In patients diagnosed with OSA, sleep stage-specific AHIs were calculated. When the ratio of AHI during REM-stage sleep $\left(\mathrm{AHI}_{\mathrm{REM}}\right)$ to AHI during non-REM-stage sleep $\left(\mathrm{AHI}_{\mathrm{NREM}}\right)$ was above $2\left(\mathrm{AHI}_{\mathrm{REM}} /\right.$ $\mathrm{AHI}_{\text {NREM }} \geq 2$ ), the patient was identified as having REM-related OSA according to previous studies [21]. All other patients were classified as having non-REM-related OSA.

\section{Sleep questionnaire}

All participants completed a set of sleep questionnaires that consisted of the Pittsburgh Sleep Quality Index (PSQI) [22], Epworth Sleepiness Scale (ESS) [23], Athens Insomnia Scale (AIS) [24], and the International Restless Legs Syndrome Rating (IRLS) scale [25].

The PSQI is a self-assessment that evaluates subjective sleep quality over the last month. The global PSQI score can range from 0 to 21 , with higher scores reflecting poorer sleep quality [22]. The ESS evaluates daytime sleepiness and consists of 8-items on chances of dozing off in different situations that are rated on a 4 -point scale ( $0=$ never, $3=$ high chance of dozing). The AIS is a self-rated measure used to evaluate insomnia severity. Two versions of this scale are used: the whole 8-item scale (AIS-8) with a total score ranging from 0 to 24 , and a brief 5 -item version (AIS- 
5) with a total score ranging from 0 to 15 , with higher scores reflecting severe insomnia. The RLS scale evaluates the severity of RLS symptoms over the past two weeks, consisting of 10 -items rated on a 5 -point scale ( $0=$ none, $4=$ very severe) with a total score ranging from 0 to 40 .

\section{Statistical analysis}

All collected data were analyzed using IBM SPSS Statistics for Windows, version 25.0 (IBM Corp., Armonk, NY, USA), and the statistical significance level was set at a p-value of $<0.05$. Comparisons between non-OSA, mild OSA, and severe OSA groups were performed using an analysis of variance, and post hoc analyses were conducted using Bonferroni's method. Independent $t$ and chi-square tests were performed to compare the demographic data, PSG parameters, and sleep questionnaires between the REMrelated OSA and non-REM-related OSA groups.

\section{RESULTS}

A total of 638 participants were included in the analyses. Among them, 464 participants were diagnosed with OSA (mild OSA, $\mathrm{n}=214$; moderate OSA, $\mathrm{n}=250$ ), and 174 participants were negative for OSA (non-OSA) based on the PSG results. A comparison of the basic demographic measures, sleep questionnaires, and PSG parameters among the three OSA severity groups are included in Table 1. These can be used to explore the tendency of these clinical variables with OSA severity.

Most of the basic demographic measures showed significant differences among the three groups. The mean ages of the non-, mild, and moderate OSA groups were 41.42 \pm 15.20 , 49.69 \pm 13.95 . $54.82 \pm 12.98$, respectively $(\mathrm{p}<0.001)$. The ratio of females was $46.6 \%$, $36.4 \%$, and $26.0 \%$ in each group ( $<<0.001)$. As the severity of OSA worsened, the mean age was significantly higher, and the ratio of females was significantly smaller. The mean weight, body mass index (BMI), and neck circumference also showed a significantly

Table 1. Comparison of basic demographic information, polysomnography parameters, and sleep questionnaires among the three participant groups classified according to the OSA severity $(n=638)$

\begin{tabular}{|c|c|c|c|c|c|}
\hline \multirow{2}{*}{ Variables } & \multirow{2}{*}{$\frac{\text { Non-OSA }(\mathrm{n}=174)}{\text { Mean } \pm \text { SD or } \mathrm{n}(\%)}$} & \multirow{2}{*}{$\frac{\text { Mild OSA }(n=214)}{\text { Mean } \pm \text { SD or } n(\%)}$} & \multirow{2}{*}{$\frac{\text { Moderate OSA }(\mathrm{n}=250)}{\text { Mean } \pm \text { SD or } \mathrm{n}(\%)}$} & \multicolumn{2}{|c|}{ Group differences } \\
\hline & & & & F or $\chi^{2}$ & p value \\
\hline \multicolumn{6}{|l|}{ Basic assessment } \\
\hline Age (yr) & $41.42 \pm 15.20^{* \dagger}$ & $49.69 \pm 13.95^{\dagger}$ & $54.82 \pm 12.98$ & 47.465 & $<0.001$ \\
\hline Sex, female & $81(46.6)^{* \dagger}$ & $78(36.4)^{\dagger}$ & $65(26.0)$ & 19.273 & $<0.001$ \\
\hline Weight (kg) & $65.27 \pm 10.89^{* \dagger}$ & $68.73 \pm 12.77$ & $70.87 \pm 11.50$ & 11.585 & $<0.001$ \\
\hline Height $(\mathrm{cm})$ & $165.92 \pm 8.04$ & $165.79 \pm 9.86$ & $166.55 \pm 9.02$ & 0.468 & 0.627 \\
\hline $\operatorname{BMI}\left(\mathrm{kg} / \mathrm{m}^{2}\right)$ & $23.64 \pm 3.11^{*+}$ & $25.01 \pm 3.57$ & $25.54 \pm 3.60$ & 15.711 & $<0.001$ \\
\hline Neck circumference, sitting $(\mathrm{cm})$ & $35.00 \pm 4.28^{*+}$ & $36.36 \pm 4.10^{\dagger}$ & $37.50 \pm 2.81$ & 23.342 & $<0.001$ \\
\hline \multicolumn{6}{|l|}{ Polysomnography } \\
\hline $\mathrm{TIB}(\min )$ & $446.25 \pm 39.71^{\dagger}$ & $441.46 \pm 39.68$ & $433.66 \pm 42.74$ & 5.155 & 0.006 \\
\hline TST (min) & $385.86 \pm 56.83^{\dagger}$ & $371.68 \pm 62.23^{\dagger}$ & $355.92 \pm 66.09$ & 12.034 & $<0.001$ \\
\hline WASO (min) & $46.53 \pm 50.46^{\dagger}$ & $57.88 \pm 52.61$ & $67.32 \pm 55.38$ & 7.871 & $<0.001$ \\
\hline SE (\%) & $86.68 \pm 11.43^{\dagger}$ & $84.31 \pm 12.54$ & $82.16 \pm 13.43$ & 6.610 & 0.001 \\
\hline $\mathrm{SL}(\mathrm{min})$ & $13.87 \pm 16.30$ & $11.91 \pm 17.59$ & $10.42 \pm 15.00$ & 2.310 & 0.100 \\
\hline $\mathrm{RL}(\min )$ & $99.25 \pm 58.97$ & $100.35 \pm 60.09$ & $102.84 \pm 61.16$ & 0.199 & 0.820 \\
\hline Total arousal index $(/ \mathrm{h})$ & $18.38 \pm 7.35^{* \dagger}$ & $22.93 \pm 9.55^{\dagger}$ & $29.55 \pm 10.56$ & 75.237 & $<0.001$ \\
\hline Stage N1 (\%) & $17.54 \pm 7.94^{* \dagger}$ & $21.35 \pm 10.53^{\dagger}$ & $27.21 \pm 11.17$ & 48.922 & $<0.001$ \\
\hline Stage N2 (\%) & $51.22 \pm 10.03^{\dagger}$ & $51.73 \pm 10.19^{\dagger}$ & $48.69 \pm 9.22$ & 6.415 & 0.002 \\
\hline Stage N3 (\%) & $11.44 \pm 8.38^{*+}$ & $7.25 \pm 6.54^{\dagger}$ & $5.75 \pm 6.53$ & 34.040 & $<0.001$ \\
\hline Stage R (\%) & $20.89 \pm 15.87^{\dagger}$ & $19.68 \pm 6.08$ & $18.36 \pm 6.89$ & 3.373 & 0.035 \\
\hline $\mathrm{AHI}_{\text {Total }}(/ \mathrm{h})$ & $2.15 \pm 1.45^{* \dagger}$ & $9.34 \pm 2.91^{\dagger}$ & $21.72 \pm 4.38$ & 1921.152 & $<0.001$ \\
\hline $\mathrm{SaO}_{2}$ nadir $(\%)$ & $91.99 \pm 2.75^{* \dagger}$ & $88.54 \pm 3.62^{\dagger}$ & $85.06 \pm 5.27$ & 143.816 & $<0.001$ \\
\hline \multicolumn{6}{|l|}{ Questionnaires } \\
\hline PSQI & $9.20 \pm 4.41^{\dagger}$ & $8.94 \pm 4.60^{\dagger}$ & $7.82 \pm 4.02$ & 6.318 & 0.002 \\
\hline ESS & $6.63 \pm 4.77$ & $6.02 \pm 4.03$ & $6.75 \pm 4.15$ & 1.851 & 0.158 \\
\hline IRLS & $4.75 \pm 8.36^{* \dagger}$ & $2.56 \pm 6.28$ & $2.91 \pm 6.36$ & 5.373 & 0.005 \\
\hline AIS-5 & $5.45 \pm 3.53^{\dagger}$ & $5.16 \pm 3.34$ & $4.59 \pm 3.48$ & 3.423 & 0.033 \\
\hline AIS-8 & $9.44 \pm 5.16^{\dagger}$ & $8.92 \pm 4.80$ & $7.86 \pm 4.85$ & 5.694 & 0.004 \\
\hline
\end{tabular}

*significantly different from mild OSA, ${ }^{\dagger}$ significantly different from moderate OSA. OSA: obstructive sleep apnea, BMI: body mass index, PSQI: Pittsburgh Sleep Quality Index, ESS: Epworth Sleepiness Scale, IRLS: the International Restless Legs Syndrome Rating scale, AIS: Athens Insomnia Scale, TIB: time in bed, TST: total sleep time, WASO: wake after sleep onset, SE: sleep efficiency, SL: sleep latency, RL: REM latency, AHI: apnea-hypopnea index, $\mathrm{SaO}_{2}$ : arterial oxygen saturation 
increasing tendency with increasing OSA severity.

From the PSG results, most of the quantitative and qualitative sleep indices showed gradual differences across the OSA severity groups. Time in bed, total sleep time, sleep efficiency, and the percentage of deep sleep (i.e., stage N3) decreased, and WASO increased as the severity of OSA worsened. Sleep latency tended to decrease with increasing OSA severity, although the difference was not statistically significant. The mean AHIs were significantly different among the groups, as expected by the group definitions. The lowest oxygen saturation (\%) and total arousal index were also significantly different according to the OSA severity. The detailed values of the PSG parameters and the statistical significance in the group differences are differentially marked in the table.

Most of the questionnaire data also showed a distinctive trend with regard to OSA severity. All participant groups reported significant sleep disturbance based on the PSQI (i.e., a cutoff score $>5$ for significant insomnia). The PSQI score was lower in the moderate OSA group compared to the less severe groups (nonOSA and mild OSA) ( $\mathrm{p}=0.002$ ). Moreover, the PSQI score tended to be higher in the less severe groups $(9.20 \pm 4.41,8.94 \pm 4.60,7.82 \pm$ 4.02; non-, mild, and moderate OSA, respectively).

The AIS score showed a similar trend. The scores of the AIS-5 and AIS-8 were highest in the non-OSA group and were significantly different from those of the more severe groups ( $\mathrm{p}=0.033$ and $\mathrm{p}=0.004$, respectively). The AIS- 5 and AIS- 8 scores showed a gradual decrease across the OSA severity groups (non-, mild, and moderate OSA; AIS-5: 5.45 $\pm 3.53,5.16 \pm 3.34,4.59 \pm 3.48$; AIS-8:
9.44 $\pm 5.16,8.92 \pm 4.80,7.86 \pm 4.85$, respectively), although this trend was not statistically significant. The IRLS score was significantly higher in the non-OSA group than in the OSA group (4.75 \pm 8.36 , $2.56 \pm 6.28,2.91 \pm 6.36, \mathrm{p}=0.005)$. The ESS scores were not significantly different among the groups.

The relationship between the subjective sleep scores and PSG parameters in the different OSA severity groups is presented in Figure 1. While the objective PSG parameters related to quality and quantity of sleep were much worse in the more severe groups, the PSQI scores became less severe as the OSA severity worsened. Therefore, a mismatch between the objective sleep data and subjective sleep perception was observed regarding OSA severity.

For additional analyses, we classified the 464 participants with mild to moderate OSA into two subgroups based on the ratio of respiratory events in the distinct sleep stages, REM and non-REM. As explained above, we employed the definition of REM-related OSA (AHIREM/AHINREM $\geq 2$ ) according to previous studies [21], with 194 participants being allocated to the REM-related OSA group. All of the other 270 participants whose OSA was not REM-related were allocated to the non-REM-related OSA group. Comparisons between the two subgroups in terms of the basic measures, questionnaires, and PSG measures are presented in Table 2.

REM-related OSA was associated with a significantly younger mean age ( $50.43 \pm 12.56$ yrs vs. $53.91 \pm 14.25 \mathrm{yrs}, \mathrm{p}=0.007)$ and a higher female ratio $(42.3 \%$ vs. $22.6 \%$, $\mathrm{p}<0.001)$ compared to nonREM-related OSA. The mean BMI of the REM-related OSA group

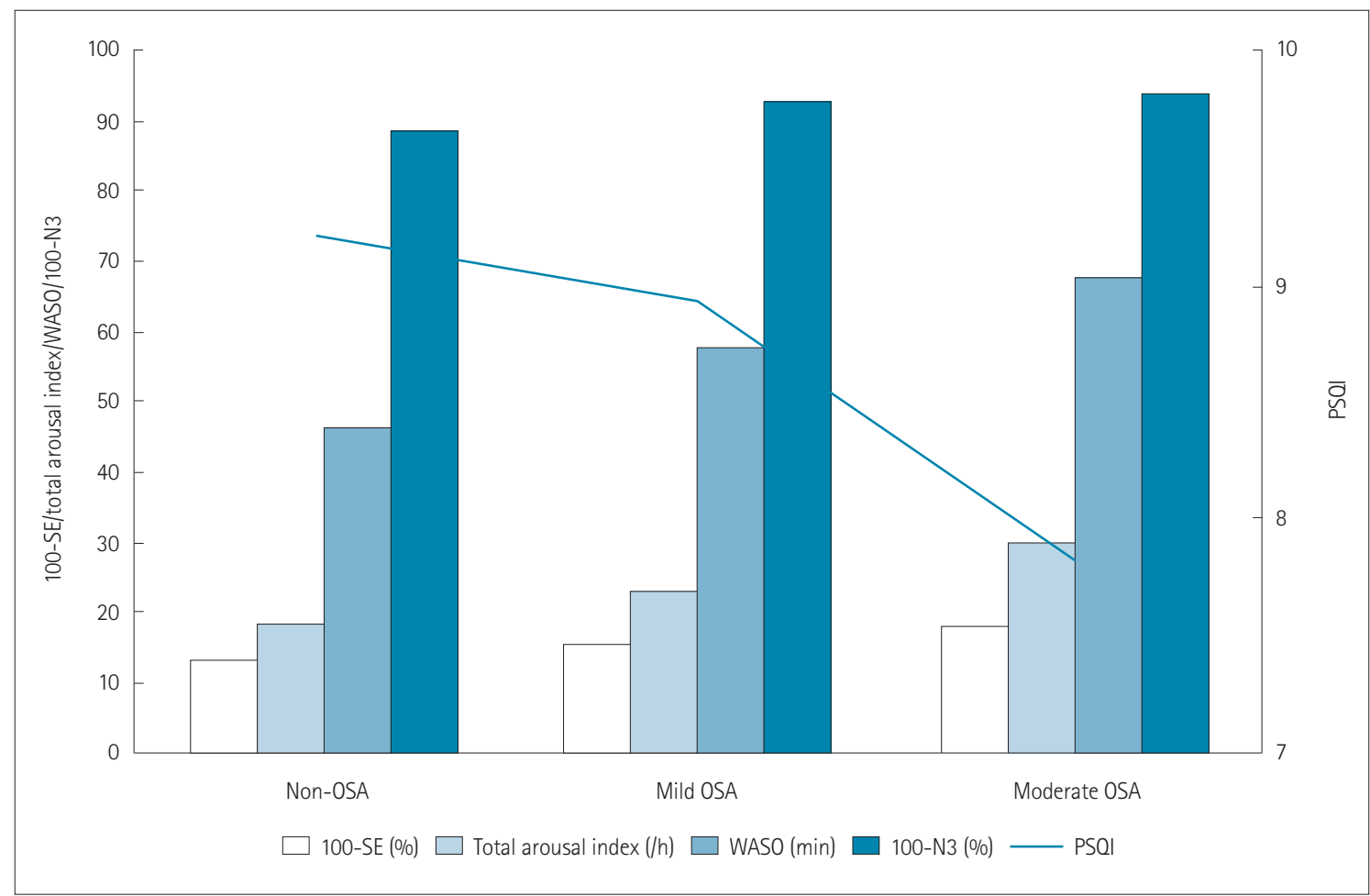

Figure 1. Objective and subjective sleep indicators derived from polysomnography and PSQI ( $n=638$ ). Non-OSA, $n=174 ;$ mild OSA, $n=214$; moderate OSA, $n=250$. The scales in the $y$-axis indicates 100-SE, total arousal index, WASO, 100 - (stage N3 proportion) (left), and the PSQI score (right). OSA: obstructive sleep apnea, SE: sleep efficiency, WASO: wake after sleep onset, N3: sleep stage N3, PSQI: Pittsburgh Sleep Quality Index. 
Table 2. Comparison of basic demographic information, polysomnography parameters, and sleep questionnaires between REM-related OSA and non-REM-related OSA $(n=464)$

\begin{tabular}{|c|c|c|c|c|}
\hline \multirow{2}{*}{ Variables } & \multirow{2}{*}{$\frac{\text { REM-related OSA }(\mathrm{n}=194)}{\text { Mean } \pm \text { SD or } \mathrm{n}(\%)}$} & \multirow{2}{*}{$\frac{\text { Non-REM-related OSA }(\mathrm{n}=270)}{\text { Mean } \pm \text { SD or } \mathrm{n}(\%)}$} & \multicolumn{2}{|c|}{ Group differences } \\
\hline & & & F or $\chi^{2}$ & $\mathrm{p}$ value \\
\hline \multicolumn{5}{|l|}{ Basic assessment } \\
\hline Age (yr) & $50.43 \pm 12.56$ & $53.91 \pm 14.25$ & 1.292 & 0.007 \\
\hline Sex, female & $82(42.3)$ & $61(22.6)$ & 20.497 & $<0.001$ \\
\hline Weight (kg) & $70.37 \pm 13.41$ & $69.53 \pm 11.14$ & 4.534 & 0.478 \\
\hline Height $(\mathrm{cm})$ & $164.16 \pm 9.74$ & $167.66 \pm 8.91$ & 4.942 & $<0.001$ \\
\hline $\mathrm{BMI}\left(\mathrm{kg} / \mathrm{m}^{2}\right)$ & $26.04 \pm 4.00$ & $24.75 \pm 3.17$ & 6.470 & $<0.001$ \\
\hline Neck circumference, sitting $(\mathrm{cm})$ & $36.62 \pm 3.24$ & $37.22 \pm 3.67$ & 0.185 & 0.069 \\
\hline \multicolumn{5}{|l|}{ Polysomnography } \\
\hline TIB (min) & $440.18 \pm 40.09$ & $435.15 \pm 42.43$ & 0.008 & 0.198 \\
\hline TST (min) & $373.78 \pm 58.32$ & $355.58 \pm 68.09$ & 3.456 & 0.003 \\
\hline WASO (min) & $55.15 \pm 47.18$ & $68.58 \pm 58.27$ & 6.394 & 0.006 \\
\hline SE (\%) & $85.00 \pm 11.27$ & $81.82 \pm 14.08$ & 8.179 & 0.007 \\
\hline $\mathrm{SL}(\min )$ & $11.26 \pm 15.13$ & $10.99 \pm 17.02$ & 0.019 & 0.858 \\
\hline $\mathrm{RL}(\min )$ & $103.40 \pm 62.19$ & $100.43 \pm 59.52$ & 0.014 & 0.604 \\
\hline Total arousal index $(/ \mathrm{h})$ & $24.22 \pm 10.35$ & $28.13 \pm 10.53$ & 0.492 & $<0.001$ \\
\hline Stage N1 (\%) & $21.51 \pm 9.82$ & $26.66 \pm 11.73$ & 2.487 & $<0.001$ \\
\hline Stage N2 (\%) & $51.95 \pm 9.56$ & $48.76 \pm 9.75$ & 0.433 & 0.001 \\
\hline Stage N3 (\%) & $7.43 \pm 6.95$ & $5.73 \pm 6.20$ & 2.338 & 0.006 \\
\hline Stage R (\%) & $19.11 \pm 6.51$ & $18.86 \pm 6.60$ & 0.001 & 0.687 \\
\hline $\mathrm{AHI}_{\text {Total }}(/ \mathrm{h})$ & $14.23 \pm 6.94$ & $17.29 \pm 7.19$ & 0.323 & $<0.001$ \\
\hline $\mathrm{SaO}_{2}$ nadir (\%) & $85.86 \pm 5.16$ & $87.24 \pm 4.62$ & 1.662 & 0.003 \\
\hline \multicolumn{5}{|l|}{ Questionnaires } \\
\hline PSQI & $9.01 \pm 4.14$ & $7.86 \pm 4.41$ & 0.012 & 0.005 \\
\hline ESS & $6.52 \pm 4.14$ & $6.34 \pm 4.09$ & 0.051 & 0.635 \\
\hline IRLS & $3.62 \pm 7.59$ & $2.13 \pm 5.15$ & 25.768 & 0.019 \\
\hline AIS-5 & $5.25 \pm 3.48$ & $4.56 \pm 3.35$ & 0.687 & 0.035 \\
\hline AIS-8 & $9.13 \pm 5.04$ & $7.79 \pm 4.64$ & 2.743 & 0.004 \\
\hline
\end{tabular}

OSA: obstructive sleep apnea, BMI: body mass index, PSQI: Pittsburgh Sleep Quality Index, ESS: Epworth Sleepiness Scale, IRLS: the International Restless Legs Syndrome Rating scale, AIS: Athens Insomnia Scale, TIB: time in bed, TST: total sleep time, WASO: wake after sleep onset, SE: sleep efficiency, SL: sleep latency, RL: REM latency, AHI: apnea-hypopnea index, $\mathrm{SaO}_{2}$ : arterial oxygen saturation

was significantly higher than that of the non-REM-related OSA group (26.04 \pm 4.00 vs. $24.75 \pm 3.17, \mathrm{p}<0.001)$, with comparable weights and significantly different heights.

REM-related OSA and non-REM-related OSA showed different PSG profiles in terms of sleep architecture and the severity of OSA. In the REM-related OSA group, the total sleep time was significantly longer (373.78 $\pm 58.32 \mathrm{~min}$ vs. $355.58 \pm 68.09 \mathrm{~min}, \mathrm{p}=$ $0.003)$, WASO was significantly shorter $(55.15 \pm 47.18 \mathrm{~min}$ vs. $68.58 \pm$ $58.27 \mathrm{~min}, \mathrm{p}=0.006$ ), and sleep efficiency was significantly higher $(85.00 \pm 11.27 \%$ vs. $81.82 \pm 14.08 \%, \mathrm{p}=0.007)$ compared to the nonREM-related OSA group. The total arousal index was significantly higher in the non-REM-related OSA group than in the REM-related OSA group ( $28.13 \pm 10.53$ vs. $24.22 \pm 10.35$, p $<0.001)$. The REMrelated OSA group had a slightly, but significantly higher proportion of stage $\mathrm{N} 3$ sleep ( $7.43 \pm 6.95 \%$ vs. $5.73 \pm 6.20 \%$, $\mathrm{p}=0.006)$, while the non-REM-related OSA group presented a higher proportion of stage N1 sleep ( $21.51 \pm 9.82 \%$ vs. $26.66 \pm 11.73 \%$, $\mathrm{p}<0.001)$. There was no statistical difference in sleep latency and the proportion of REM sleep between the groups.
The AHI of REM-related OSA was less severe than that of nonREM-related OSA (14.23 \pm 6.94 vs. 17.29 \pm 7.19 , p<0.001). However, the lowest oxygen saturation of the REM-related OSA group was significantly lower than that of the non-REM-related OSA group (85.86 $\pm 5.16 \%$ vs. $87.24 \pm 4.62 \%, \mathrm{p}<0.001)$.

According to the questionnaire data, the REM-related OSA group reported significantly higher scores in the PSQI $(9.01 \pm 4.14$ vs. $7.86 \pm 4.41, \mathrm{p}=0.005)$, IRLS ( $3.62 \pm 7.59$ vs. $2.13 \pm 5.15, \mathrm{p}=0.019)$, and AIS (AIS-5, $5.25 \pm 3.48$ vs. $4.56 \pm 3.35, \mathrm{p}=0.035$; AIS-8, $9.13 \pm$ 5.04 vs. $7.79 \pm 4.64, \mathrm{p}=0.004$ ) than the non-REM-related OSA group. The ESS did not differ significantly between the two groups.

A further subgroup analysis conducted only on the male participants with OSA $(n=321)$ showed similar results. The participants in the REM-related OSA group $(n=112)$ presented better PSG profiles than those in non-REM-related OSA $(n=209)$ in terms of total sleep time, sleep efficiency, WASO, the proportion of each sleep stage, and the total arousal index (data not shown). In contrast, the REM-related OSA group reported significantly higher scores in the PSQI $(8.46 \pm 3.93$ vs. $7.39 \pm 4.26, \mathrm{p}=0.027)$ and 
AIS-8 (8.71 \pm 4.93 vs. $7.30 \pm 4.39, \mathrm{p}=0.010)$ compared to the nonREM-related OSA group.

\section{DISCUSSION}

This study aimed to explore sleep perception in patients with OSA. To investigate the association between SSM and OSA, we compared the degree of subjective-objective sleep discrepancies among the participant groups of different OSA severities. We also examined the differences in sleep discrepancies between REMrelated and non-REM-related OSA groups. This was a retrospective study using a large sample consisting of patients who underwent PSG at the sleep disorder clinic of a university hospital under diagnostic suspicion of OSA. Here, we included patients with or without OSA of a mild to moderate degree to focus specifically on subjective sleep perception by excluding severe OSA.

The main comparison analyses showed that most of the PSG indices on sleep architecture and sleep quality were in accordance with the severity of OSA. That is, participants with higher AHI values presented a much poorer sleep quality with significantly shorter total sleep time, lower sleep efficiency, longer WASO, more arousal, and less amount of deep sleep. This is consistent with the widely acknowledged fact that OSA causes sleep fragmentation and prevents deep sleep, depending on its severity. However, interestingly, the subjective perception of sleep disturbance did not match the OSA severity. In particular, the participants who were negative for OSA reported the most severe sleep complaints next to the mild OSA participants, and those in the most severe group (i.e., moderate OSA) reported the least sleep complaints based on multiple sleep questionnaires. This indicates that there is a significant mismatch between subjective and objective assessments of sleep state in individuals with OSA.

All groups in this study presented significant subjective sleep disturbance with global PSQI scores above the cutoff. While the impact of OSA is expected to be directly responsible for subjective sleep disorders in the groups with mild and moderate OSA, the participants in the non-OSA group can be diagnosed with paradoxical insomnia. Paradoxical insomnia is an extreme case of SSM with no explainable objective findings [5]. They presented all respiratory event-related parameters within the normal ranges, excluding the diagnosis of sleep-disordered breathing. They were found to have a sleep efficiency above $85 \%$, sleep latency below 30 minutes, total sleep time around 6.5 hours, preserved deep sleep, and intact sleep architecture in PSG, which indicates fair quality and quantity of sleep. However, they showed slightly longer sleep latency than the other groups (although not significantly different), and slightly increased arousal (18.38/h) in the absence of respiratory event-related arousals. As subjective complaints, they reported higher scores even in ESS and IRLS in addition to the PSQI and AIS.

These participants with paradoxical insomnia may be accompanied by physiological changes such as heightened sympathetic tone or conditioned cortical arousal. Although not assessed in this study, personality factors (i.e., neuroticism) or current psychopathology (i.e., depression) may have also implicated SSM, as suggested in previous studies $[7,8]$ It should be noted that these participants were initially suspected of having OSA rather than psychophysiological insomnia according to their clinical evaluation, which includes the patient history, a physical exam, response to sedative medications, and comorbid medical illnesses. This shows that it can be challenging to differentiate between SSM and OSA.

Regarding the participants with mild and moderate OSA, excessive daytime sleepiness was severe in the more severe OSA group. However, the PSQI was significantly higher in the milder group, and AIS also tended to be higher in the milder group. As suggested in previous literature, adaptive effort to maintain sleep may change the threshold of arousal in OSA [26]. Our finding may also suggest that when the severity of OSA aggravates to a certain degree, the patient may not perceive their night sleep disturbance and even underestimate it. Moreover, among the patient-reported information, subjective reports on daytime functioning, such as excessive sleepiness, may better reflect the severity of OSA, rather than their report on night sleep. In addition, it needs to be noticed that individuals with paradoxical insomnia also tend to exaggerate excessive sleepiness and fatigue.

This finding is consistent with previous studies that showed significant sleep discrepancies in OSA [16] and a higher prevalence of subjective insomnia in milder OSA $[27,28]$. The current finding is partially in line with studies that suggested no association between SSM (i.e., exaggeration of sleep disturbance) and OSA $[17,18]$. We demonstrated that patients with more severe OSA tended to be less able to perceive sleep disturbance. Some researchers termed this phenomenon positive sleep discrepancy [5]. Nevertheless, our findings conflict with an earlier observation in which patients with OSA reported significantly shorter total sleep time and longer sleep latency compared to those without OSA, suggesting that OSA is a contributing factor for SSM [13]. Our findings may have been affected by selection bias, as Krell and Kapur [27] explained, since the patients who were referred to the university-affiliated hospital have higher probabilities of multiple comorbid sleep disorders or severe, refractory subjective complaints.

In the additional analyses, we demonstrated that patients with REM-related OSA tend to present more discrepancies between subjective and objective sleep compared to those with non-REMrelated OSA. REM-related OSA refers to the OSA in which apnea and hypopnea occur predominantly or exclusively during REM sleep and account for 12-36\% of OSA [29,30]. Since REM-related OSA is suggested to have distinct clinical characteristics and effects compared to non-REM-related OSA, we conducted an additional analysis to investigate whether REM-specific OSA characteristics are associated with SSM in OSA. The results of this analysis showed that the REM-related OSA group reported significantly higher subjective sleep complaints on all evaluated sleep scales and better objective sleep parameters. Furthermore, a less severe 
degree of OSA was recorded in PSG compared to the non-REMrelated OSA group. Therefore, from our findings, the sleep stage characteristics are suggested to be an important factor for SSM in OSA. It is of note that the lowest oxygen saturation was the single index that was more severe in REM-related OSA. It can be assumed that the degree of oxygen desaturation may contribute to sleep perception, particularly when associated with the REMstage.

This finding is consistent with a previous study [21] in which significantly higher PSQI was observed in patients with REMrelated OSA, especially females. The current finding is contrary to the report of Chami et al. [31], in which no significant differences in various sleep scales were found between patients with REM- and non-REM-related OSA. Our finding on the comparison of REM- and non-REM-related OSA supports earlier reports in which REM-related OSA was associated with a higher female prevalence, younger age, milder severity of OSA [32], and a larger proportion of deep sleep [33].

Since 'female' and 'insomnia' were reported to be significant risk factors for both SSM [13] and REM-related OSA [21,32], we conducted an additional subgroup analysis on the male subsample. The findings of a larger sleep mismatch in REM-related OSA were also observed in the male participants with OSA. This supports our suggestion that REM-related OSA is a risk factor for SSM in OSA.

This study has several limitations. First, the PSG data used in the analyses reflect the sleep state of a single night, while the sleep questionnaires assessed subjective sleep over a week. However, since monitoring PSG over a certain period is practically impossible, this would be a difficult problem to solve when investigating SSM. The use of actigraphy may be considered in the future study. Second, we used the global score of sleep scales to assess subjective sleep complaints instead of subjective estimates of each sleep index, such as subjective sleep latency and total sleep time. However, the global PSQI score is the result scored from each assessment item, including subjective sleep latency, total sleep time, WASO, and sleep efficiency. Moreover, we assessed multiple sleep scales to better reflect subjective sleep complaints. Third, we did not consider the effects of major psychiatric disorders, medical comorbidities, and other comorbid sleep disorders. Our results need to be replicated in future research, with better control of potential confounders such as Beck Depression Inventory, Beck Anxiety Inventory or periodic limb movement index.

In conclusion, our findings demonstrate different degrees of significant mismatch between subjective and objective sleep states in individuals with non-, mild, and moderate OSA. This is the largest-scale study, as far as we are aware, to evaluate sleep perception in OSA. This is also the first to report preliminary evidence of REM-related OSA as a contributing factor of SSM in OSA. The diagnosis of sleep disorders can be difficult due to the misperception of sleep states. Recognition of significant sleep mismatch is clinically important so that patients with sleep disorders can be properly treated. Future research is warranted to replicate the results of the current study and expand our understanding of sleep perception in OSA.

\section{Acknowledgments}

This study was supported by the Korea Health 21 R\&D Project funded by the National Research Foundation of Korea (2016M3 C7A1904345, 2017M3A9F1031220, and 2019R1A2C2084158).

\section{Conflicts of Interest}

The authors have no potential conflicts of interest to disclose.

\section{Author Contributions}

Conceptualization: Heon-Jeong Lee, Sehyun Jeon, Joon-Ho Lee. Methodology: Heon-Jeong Lee, Sehyun Jeon, Joon-Ho Lee. Data curation: Joon-Ho Lee, Sehyun Jeon. Formal analysis: Joon-Ho Lee. Investigation: Joon-Ho Lee. Funding acquisition: Heon-Jeong Lee. Supervision: Heon-Jeong Lee, Sehyun Jeon. Validation: HeonJeong Lee, Sehyun Jeon. Visualization: Heon-Jeong Lee, Sehyun Jeon. Writing - original draft: Sehyun Jeon, Joon-Ho Lee. Writing_-review \& editing: Heon-Jeong Lee, Sehyun Jeon.

\section{ORCID iDs}

Sehyun Jeon (1)

https://orcid.org/0000-0002-6594-6556

Joon-Ho Lee (10

https://orcid.org/0000-0002-5873-4598

\section{REFERENCES}

1. Harvey AG, Tang NK. (Mis)perception of sleep in insomnia: a puzzle and a resolution. Psychol Bull 2012;138:77-101.

2. Bastien CH, Ceklic T, St-Hilaire P, Desmarais F, Pérusse AD, Lefrançois J, et al. Insomnia and sleep misperception. Pathol Biol (Paris) 2014;62:241-251.

3. American Academy of Sleep Medicine. International classification of sleep disorders. 3rd ed. Darien, IL: American Academy of Sleep Medicine, 2014.

4. An H, Chung S. A case of obstructive sleep apnea syndrome presenting as paradoxical insomnia. Psychiatry Investig 2010;7:75-78.

5. Rezaie L, Fobian AD, McCall WV, Khazaie H. Paradoxical insomnia and subjective-objective sleep discrepancy: a review. Sleep Med Rev 2018;40: 196-202.

6. Castelnovo A, Ferri R, Punjabi NM, Castronovo V, Garbazza C, Zucconi M, et al. The paradox of paradoxical insomnia: a theoretical review towards a unifying evidence-based definition. Sleep Med Rev 2019;44:70-82.

7. Dorsey CM, Bootzin RR. Subjective and psychophysiologic insomnia: an examination of sleep tendency and personality. Biol Psychiatry 1997;41: 209-216.

8. Vanable PA, Aikens JE, Tadimeti L, Caruana-Montaldo B, Mendelson WB. Sleep latency and duration estimates among sleep disorder patients: variability as a function of sleep disorder diagnosis, sleep history, and psychological characteristics. Sleep 2000;23:71-79.

9. Feige B, Baglioni C, Spiegelhalder K, Hirscher V, Nissen C, Riemann D. The microstructure of sleep in primary insomnia: an overview and extension. Int J Psychophysiol 2013;89:171-180.

10. Hermans LWA, Leufkens TR, van Gilst MM, Weysen T, Ross M, Anderer P, et al. Sleep EEG characteristics associated with sleep onset misperception. Sleep Med 2019;57:70-79.

11. Feige B, Al-Shajlawi A, Nissen C, Voderholzer U, Hornyak M, Spiegelhalder K, et al. Does REM sleep contribute to subjective wake time in primary insomnia? A comparison of polysomnographic and subjective sleep in 100 patients. J Sleep Res 2008;17:180-190.

12. St-Jean G, Turcotte I, Pérusse AD, Bastien CH. REM and NREM power 
spectral analysis on two consecutive nights in psychophysiological and paradoxical insomnia sufferers. Int J Psychophysiol 2013;89:181-194.

13. Hwang KJ, Lee JS, Son HJ, Kim KH, Lee MJ, Kim J. Factors related to sleep time underestimation in obstructive sleep apnea: retrospective study based on polysomnographic data. J Korean Neurol Assoc 2019;37:144-149.

14. Chervin RD. Sleepiness, fatigue, tiredness, and lack of energy in obstructive sleep apnea. Chest 2000;118:372-379.

15. McNicholas WT, Ryan S. Obstructive sleep apnoea syndrome: translating science to clinical practice. Respirology 2006;11:136-144.

16. Nam H, Lim JS, Kim JS, Lee KJ, Koo DL, Lee C. Sleep perception in obstructive sleep apnea: a study using polysomnography and the multiple sleep latency test. J Clin Neurol 2016;12:230-235.

17. Pinto LR Jr, Pinto MC, Goulart LI, Truksinas E, Rossi MV, Morin CM, et al. Sleep perception in insomniacs, sleep-disordered breathing patients, and healthy volunteers--an important biologic parameter of sleep. Sleep Med 2009;10:865-868.

18. Bianchi MT, Williams KL, McKinney S, Ellenbogen JM. The subjective-objective mismatch in sleep perception among those with insomnia and sleep apnea. J Sleep Res 2013;22:557-568.

19. Choi SJ, Suh S, Ong J, Joo EY. Sleep misperception in chronic insomnia patients with obstructive sleep apnea syndrome: implications for clinical assessment. J Clin Sleep Med 2016;12:1517-1525.

20. Berry RB, Budhiraja R, Gottlieb DJ, Gozal D, Iber C, Kapur VK, et al. Rules for scoring respiratory events in sleep: update of the 2007 AASM Manual for the scoring of sleep and associated events. Deliberations of the Sleep Apnea Definitions Task Force of the American Academy of Sleep Medicine. J Clin Sleep Med 2012;8:597-619.

21. Hoshino T, Sasanabe R, Murotani K, Hori R, Mano M, Nomura A, et al. Insomnia as a symptom of rapid eye movement-related obstructive sleep apnea. J Clin Med 2020;9:1821.

22. Buysse DJ, Reynolds CF 3rd, Monk TH, Berman SR, Kupfer DJ. The Pittsburgh Sleep Quality Index: a new instrument for psychiatric practice and research. Psychiatry Res 1989;28:193-213.

23. Johns MW. A new method for measuring daytime sleepiness: the Epworth sleepiness scale. Sleep 1991;14:540-545.

24. Soldatos CR, Dikeos DG, Paparrigopoulos TJ. The diagnostic validity of the Athens Insomnia Scale. J Psychosom Res 2003;55:263-267.

25. Walters AS, LeBrocq C, Dhar A, Hening W, Rosen R, Allen RP, et al. Validation of the International Restless Legs Syndrome Study Group rating scale for restless legs syndrome. Sleep Med 2003;4:121-132.

26. Zinchuk AV, Gentry MJ, Concato J, Yaggi HK. Phenotypes in obstructive sleep apnea: a definition, examples and evolution of approaches. Sleep Med Rev 2017;35:113-123.

27. Krell SB, Kapur VK. Insomnia complaints in patients evaluated for obstructive sleep apnea. Sleep Breath 2005;9:104-110.

28. So SJ, Lee HJ, Kang SG, Cho CH, Yoon HK, Kim L. A comparison of personality characteristics and psychiatric symptomatology between upper airway resistance syndrome and obstructive sleep apnea syndrome. Psychiatry Investig 2015;12:183-189.

29. Conwell W, Patel B, Doeing D, Pamidi S, Knutson KL, Ghods F, et al. Prevalence, clinical features, and CPAP adherence in REM-related sleep-disordered breathing: a cross-sectional analysis of a large clinical population. Sleep Breath 2012;16:519-526.

30. Mano M, Hoshino T, Sasanabe R, Murotani K, Nomura A, Hori R, et al. Impact of gender and age on rapid eye movement-related obstructive sleep apnea: a clinical study of 3234 Japanese OSA patients. Int J Environ Res Public Health 2019;16:1068.

31. Chami HA, Baldwin CM, Silverman A, Zhang Y, Rapoport D, Punjabi NM, et al. Sleepiness, quality of life, and sleep maintenance in REM versus nonREM sleep-disordered breathing. Am J Respir Crit Care Med 2010;181: 997-1002.

32. Campos-Rodríguez F, Fernández-Palacín A, Reyes-Núñez N, ReinaGonzález A. [Clinical and polysomnographic features of rapid-eye-movement-specific sleep-disordered breathing]. Arch Bronconeumol 2009;45: 330-334.

33. Haba-Rubio J, Janssens JP, Rochat T, Sforza E. Rapid eye movement-related disordered breathing: clinical and polysomnographic features. Chest 2005; 128:3350-3357. 